\section{RMD Open}

Rheumatic \&

Musculoskeletal Diseases

\title{
Malignancy incidence in 5294 patients with juvenile arthritis
}

Omid Zahedi Niaki, ${ }^{1}$ Ann E Clarke, ${ }^{2}$ Rosalind Ramsey-Goldman, ${ }^{3}$ Rae Yeung, ${ }^{4}$ Kristen Hayward, ${ }^{5}$ Kiem Oen, ${ }^{6}$ Ciarán M Duffy, ${ }^{7}$ Alan Rosenberg, ${ }^{8}$ Kathleen M O'Neil, ${ }^{9}$ Emily von Scheven, ${ }^{10}$ Laura Schanberg, ${ }^{11}$ Jeremy Labrecque, ${ }^{1}$ Shirley M L Tse, ${ }^{4}$ Rachana Hasija, ${ }^{4}$ Jennifer L F Lee, ${ }^{1}$ Sasha Bernatsky ${ }^{1,12}$

\section{ABSTRACT}

Clarke AE, RamseyGoldman R, et al. Malignancy incidence in 5294 patients with juvenile arthritis. $R M D$ Open 2016;2:e000212. doi:10.1136/rmdopen-2015000212

- Prepublication history for this paper is available online. To view these files please visit the journal online (http://dx.doi.org/10.1136/ rmdopen-2015-000212).

Received 16 November 2015 Revised 8 March 2016 Accepted 9 March 2016
CrossMark

For numbered affiliations see end of article.

Correspondence to Dr Sasha Bernatsky; Sasha.bernatsky@mcgill.ca
Objective: To determine cancer incidence in a large clinical juvenile-onset arthritis population.

Methods: We combined data from 6 existing North American juvenile-onset arthritis cohorts. Patients with juvenile-onset arthritis were linked to regional cancer registries to detect incident cancers after cohort entry, defined as first date seen in the paediatric rheumatology clinic. The expected number of malignancies was obtained by multiplying the personyears observed (defined from cohort entry to end of follow-up) by the geographically matched age, sex and calendar year-specific cancer rates. The standardised incidence ratios (SIR; ratio of cancers observed to expected) were generated, with $95 \% \mathrm{Cls}$.

Results: The 6 juvenile arthritis registries provided a total of 5294 patients. The mean age at cohort entry was 8.9 (SD 5.0) years and $68 \%$ of participants were female. The mean duration of follow-up was 6.8 years with a total of 36063 person-years spanning 19782012. During follow-up, 9 invasive cancers occurred, compared with 10.9 expected (SIR 0.82, 95\% Cl 0.38 to 1.5). 3 of these were haematological (Hodgkin's, non-Hodgkin's lymphoma and leukaemia). 6 of the patients with cancer were exposed to diseasemodifying drugs; 5 of these had also been exposed to biological agents.

Conclusions: We did not clearly demonstrate an increase in overall malignancy risk in patients with juvenile-onset arthritis followed for an average of almost 7 years. 3 of the 9 observed cancers were haematological. 5 of the cancers arose in children exposed to biological agents. Longer follow-up of this population is warranted, with further study of drug effects.

\section{INTRODUCTION}

Several reports have been published over the past decade examining malignancy risk in rheumatic disease populations. Few studies have specifically reported on malignancy occurrence in juvenile arthritis, and the majority of these reports are based on analysis of administrative data sets. The objective of our study was to determine malignancy

\section{Key messages}

What is already known about this subject?

- In many systemic autoimmune rheumatic diseases, including rheumatoid arthritis, there is an increased risk (compared to the general population) of certain malignancies.

What does this study add?

- This study provides a multi-centre perspective of the issue of cancer risk in juvenile arthritis, in clinic-based settings.

How might this impact on clinical practice?

- In juvenile-onset arthritis, cancer was a relatively rare event, which is reassuring for patients and physicians. However, the possibility of an increased risk of hematological malignancies in juvenile-onset arthritis remains a concern. This and the effects of drug exposures, should be the focus of future studies.

incidence, using cancer registry linkage, in a large population of juvenile-onset arthritis followed at six North American paediatric centres.

\section{MATERIALS AND METHODS}

We combined data from four paediatric rheumatology clinics in Canada and two in the USA (table 1). Seattle's cohort was based on an electronic medical record search, and all other cohort participants were enrolled from paediatric rheumatology clinics at the time they met clinical criteria for juvenile-onset arthritis. Thus, the cohorts were independently created by each local investigator, and ethics permission was obtained for cancer registry linkage in order to ascertain this outcome across all cohorts. We then pooled the data for our study.

Patients were linked to their regional (provincial or state) cancer registries to detect incident observed cancers occurring after 
Table 1 List of participating cohorts

\begin{tabular}{|c|c|c|c|c|}
\hline Centre & $\mathbf{N}$ & $\begin{array}{l}\text { Observation } \\
\text { period }\end{array}$ & $\begin{array}{l}\text { Enrolment } \\
\text { period }\end{array}$ & Inclusion criteria \\
\hline $\begin{array}{l}\text { Children's Hospital, Health Sciences Centre, } \\
\text { Winnipeg, Manitoba }\end{array}$ & 816 & 1999-2008 & 1974-2007 & ILAR \\
\hline $\begin{array}{l}\text { Royal University Hospital, Saskatoon, } \\
\text { Saskatchewan }\end{array}$ & 364 & 1995-2009 & $1981-2011$ & $\begin{array}{l}\text { 1981-1998: ACR } \\
\text { 1999-2011: ILAR }\end{array}$ \\
\hline $\begin{array}{l}\text { The Toronto Hospital for Sick Children, } \\
\text { Toronto, Ontario }\end{array}$ & 1722 & 1978-2012 & 1974-2013 & ILAR \\
\hline $\begin{array}{l}\text { The Montreal Children's Hospital, Montreal, } \\
\text { Quebec }\end{array}$ & 656 & 1995-2006 & $1991-2010$ & ILAR \\
\hline $\begin{array}{l}\text { The Seattle Children's Hospital, Seattle, } \\
\text { Washington }\end{array}$ & 1602 & 2003-2011 & 2003-2012 & $\begin{array}{l}\text { Administrative database data } \\
\text { using ICD- } 9 \text { codes }\end{array}$ \\
\hline $\begin{array}{l}\text { The Children's Hospital at Oklahoma } \\
\text { University Medical Center, Oklahoma }\end{array}$ & 134 & 1999-2008 & 1996-2011 & $\mathrm{ACR}$ \\
\hline Total & 5294 & & & \\
\hline
\end{tabular}

cohort entry, defined as date first seen in the paediatric rheumatology clinic, for all patients. The end of follow-up was defined by the date of cancer registry linkage data, thus allowing the observation time for participants to include not only childhood and adolescence but also adulthood. The expected number of malignancies was obtained by multiplying the person-years in the cohort (defined from cohort entry to end of follow-up) by the age, sex and calendar year-specific general population incidence rates from each cancer registry, and summing across person-years of observation, across all cohorts. The observed and expected events were therefore both derived from cancer registry sources.

The total number of observed cancers was pooled across all studies. The standardised incidence ratio (SIR; ratio of cancers observed to expected) was generated (for overall malignancy and for haematological malignancies), along with $95 \%$ CIs, based on the assumption of cancer occurrence as a Poisson-distributed variable. Results were also stratified by sex and age group of person-time (categorised into 0 to 19 and $\geq 20$ years of age), as well as calendar year period (before and after the year 2000). Cancer cases were described in terms of demographics and arthritis subtype. Over the entire calendar period (beginning in 1978, see table 1), the nomenclature of juvenile arthritis evolved, from juvenile rheumatoid arthritis (JRA, with three subcategories) to juvenile idiopathic arthritis (JIA, with seven categories). Enthesitis-related arthritis, psoriatic arthritis and undifferentiated arthritis were included in the juvenile arthritis classification system only after $2004 .{ }^{1}$ Accordingly, these subtypes were only included in our data after this date. For the purpose of clarity, the term juvenile-onset arthritis' is used to refer to the patients in our study, as opposed to the terms 'JRA' or 'JIA'.

\section{RESULTS}

Our study included 5294 patients with juvenile-onset arthritis. The mean age at cohort entry was 8.9 (SD 5.0) years and $68 \%$ of the participants were female. The most common race/ethnicity was Caucasian. The most common arthritis subtype was oligoarticular or pauciarticular $(\mathrm{N}=1862,35 \%)$. The mean duration of follow-up was 6.8 years, resulting in a total observation period of 36063 years spanning the calendar period (with the majority (76\%) of the person-years being after 2000).

During the follow-up period under study, 9 invasive cancers were recorded, compared with 10.9 expected (SIR $0.82,95 \%$ CI 0.38 to 1.5 ). Sensitivity analyses removing the Seattle cohort, or restricting to Canadian-only cohort, showed similar results (data not shown). Six cancer cases were female (table 2), and the mean age at cancer diagnosis was 17 years (SD 6, minimum 9 and maximum 30 years). At the time of cancer diagnosis, the average time since arthritis diagnosis was 7 years (SD 6, minimum 2 and maximum 19 years).

Three of the cancers were haematological (one each of Hodgkin's lymphoma, hepatosplenic T-cell non-Hodgkin's lymphoma, B-cell lymphoid leukaemia) and a fourth cancer was a post-transplant lymphoproliferative disorder that was malignant and non-remitting, resulting in death. The SIR specific for haematological malignancies (not including the post-transplant lymphoproliferative disorder) was 1.25 (95\% CI 0.26 to 3.66). The T-cell non-Hodgkin's lymphoma case had received etanercept in the past, but never azathioprine, and the malignancy developed while the patient was on anakinra and methotrexate. The patient with a post-transplant lymphoproliferative disorder had been on methotrexate, etanercept, cyclophosphamide and rituximab, prior to the transplant, and mycophenolate and tacrolimus thereafter. Non-haematological cancers included one each of glioma, primitive neuroectodermal (Ewing) cancer, renal cell adenocarcinoma, papillary follicular adenocarcinoma thyroid cancer and endometrial adenocarcinoma. Characteristics of each case are presented in table 2. 
Table 2 Characteristics of cancers in juvenile-onset arthritis

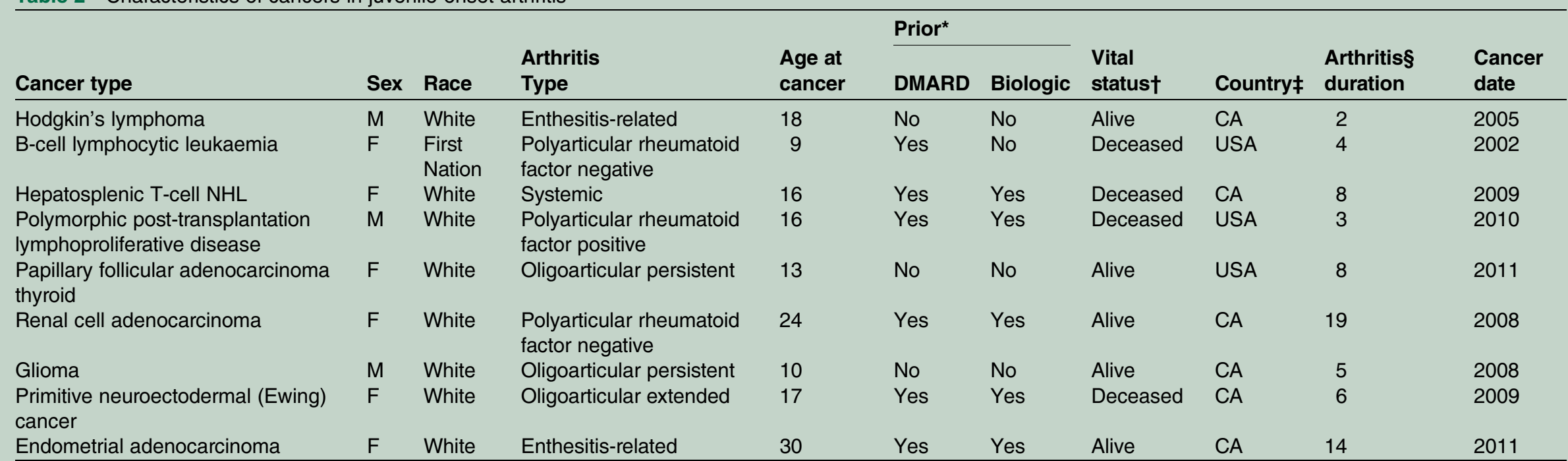

${ }^{*}$ Prior DMARD=use of disease-modifying antirheumatic agent prior to cancer onset.

†Vital status as of time of cancer registry linkage.

$¥ \mathrm{CA}=$ Canada.

$\S$ Time between arthritis diagnosis and cancer diagnosis.

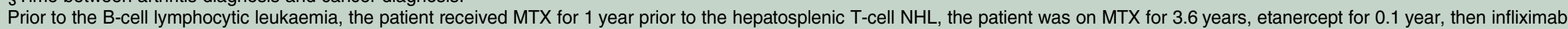
for 1.7 years, then anakinra for 1.9 years. Prior to the post-transplant lymphoproliferative disorder, the patient had been on MTX for 1 month, etanercept for 6 months, cyclophosphamide for

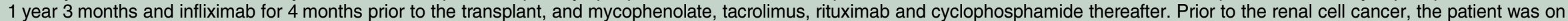

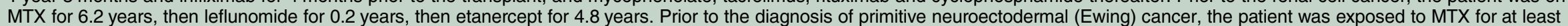

20 months and leflunomide for 3 months, and was prescribed Enbrel but was not compliant.

DMARD, disease-modifying antirheumatic drug; F, female; M, male; MTX, methotrexate; NHL, non-Hodgkin's lymphoma. 
The overall cancer SIR for females was 0.77 (95\% CI 0.28 to 1.67 ) and for males, 0.97 (95\% CI 0.20 to 2.83). When stratifying SIR estimates by age group of the person-time, in the younger age group of person-time (person-time contributed from 0 to 19 years of age), the SIR was 1.61 (95\% CI 0.65 to 3.33) for overall cancers and 1.91 (95\% CI 0.39 to 5.59) for haematological cancers. The overall SIR for patient-years in the age group $\geq 20$ years was 0.30 (95\% CI 0.04 to 1.09 ); no haematological cancers occurred during patient-years accumulated in the age group $\geq 20$ years. The SIRs divided calendar year period showed that no cancers had occurred up to the year 2000 (a maximum of two might have been expected), and from 2001 onward, the SIR was 1.01 (95\% CI 0.46 to 1.92 ).

\section{DISCUSSION}

Our study is one of the few studies examining malignancy risk in clinically confirmed juvenile-onset arthritis. Comparing the cancer events in over 5000 patients with juvenile-onset arthritis (36063 person-years) with the appropriate regional age-matched and sex-matched general population cancer rates, we were unable to establish an increased malignancy incidence in the juvenile-onset arthritis group. However, we cannot rule out an increased risk, particularly for haematological malignancies, when person-time is limited to $0-19$ years of age.

Two-thirds (six of nine) of the malignancies in the cohort occurred in individuals who had been exposed to disease-modifying antirheumatic drugs (DMARDs) and/ or biological agents (five individuals being exposed to both). Although we did not have exposure data on all individuals, cohort directors estimate that about 50-60\% of all cohort participants would have been on DMARDs, and $10-15 \%$ on biologics.

Other groups have attempted to ascertain the baseline cancer risk in juvenile arthritis, but these have primarily used administrative data sets, where the arthritis diagnosis was not clinically confirmed, and the cancer diagnosis often ascertained through administrative claims, not through actual cancer registry linkage. The largest such study used US Medicaid data to identify children $(\mathrm{n}=7812)$ with billing codes that might represent arthritis (including rheumatoid arthritis (RA), psoriatic arthritis, ankylosing spondylitis and inflammatory bowel disease-associated arthritis). The billing code algorithm was not clinically validated. This arthritis cohort was compared with a cohort of children with asthma ( $\mathrm{N}=652$ 234) and attention-deficit/hyperactivity (ADHD, $\mathrm{N}=321821) .{ }^{2}$ In this study, cancer outcomes were identified using an adapted version of a previously validated malignancy-finding algorithm of the International Classification of Diseases, Ninth Revision (ICD-9) codes, procedure codes and pharmacy claims. ${ }^{3}$ Malignancies were not clinically confirmed. For all children with arthritis compared with those of the ADHD cohort, the SIR was 4.4 (95\% CI 1.8 to 9.0$)$ for overall malignancies. The SIR for children exposed to neither methotrexate nor biological therapies was 6.9 (95\% CI 2.3 to 16). The authors therefore suggest that there is an increased baseline risk of malignancy in juvenile arthritis. A clear limitation of this study, however, is that the cancer events (and none of the arthritis diagnoses) were not actually clinically confirmed.

Similarly, Nordstrom et $a l^{4}$ used a large national US claims database to identify a cohort $(\mathrm{N}=3605)$ of children with ICD-9 RA billing codes, and compared them with children without ICD RA billing codes (37 689 individuals). Again, neither the arthritis diagnoses nor cancer events were actually clinically confirmed. The internal comparison suggested an increased risk of malignancy in the juvenile arthritis cohort (HR 2.8, CI 0.9 to 8.3 ). Interestingly, in this study, cancer events in the non-arthritis cohort were $40 \%$ above the US Surveillance, Epidemiology, and End-Results general population cancer rates, suggesting that cancer events identified through claims data may have been overestimated. Although the authors did not feel this was a likely bias, it may be particularly important since children with arthritis presumably have more physician visits and hospitalisations than children without arthritis, and hence those children would have more opportunities to have a cancer falsely identified via this approach.

Finally, the most recent study on the topic examined malignancy risk in 2892 Taiwanese juvenile arthritis participants. ${ }^{5}$ Once more, the study was based on administrative data, and the arthritis diagnoses and cancer events were not actually clinically confirmed. The authors found an increased risk of malignancy in patients with juvenile arthritis who were naïve to methotrexate and biologics (adjusted HR=3.14, 95\% CI 1.98 to 4.98). The relative risk (RR) for malignancy in patients with juvenile arthritis treated with methotrexate was 2.02 (95\% CI 0.67 to 6.04 ), and in patients treated with antitumour necrosis factor (TNF) agents, the RR was 2.07 (95\% CI 0.36 to 11.49 ). Haematological malignancies appeared to make up a significant component of the increased cancer risk seen in that study. The conclusion of the authors was that, seemingly, neither methotrexate nor anti-TNF biologics increased the risk of cancer in juvenile arthritis, beyond the baseline risk.

Our results differ somewhat from the last three aforementioned cohort studies. A potential reason for this is that the other studies did not clinically confirm the juvenile arthritis diagnoses, but even more importantly, in the aforementioned studies, the cancer outcome was not verified by cancer registry linkage. Our juvenile arthritis cohort data, using cancer registry linkage as the 'gold standard' of cancer outcome ascertainment, correlate with conclusions drawn from adult patients with RA: no significant increase in overall baseline cancer risk. In adults with RA, there is an increased incidence of haematological cancers, and though our data were not conclusive in this regard, there may have been a 
potential trend for increased incidence of haematological cancers in our paediatric arthritis cohort.

Conclusions similar to ours can be drawn from two European studies of juvenile-onset arthritis, which also used cancer registry linkage to identify malignancies. One study focused primarily on patients with RA and osteoarthritis but also included a cohort $(n=896)$ of patients diagnosed as having juvenile-onset arthritis. ${ }^{6}$ In patients with RA, the overall cancer risk was comparable to that of the general population whereas the risk of developing haematological malignancies was increased by nearly twofold. In patients with juvenile-onset arthritis, only four malignancies were identified and no increase was observed in the overall cancer rate. The second European study ${ }^{7}$ used nationwide Swedish Patient Registry data to identify 9020 juvenile arthritis and 44858 general population comparator patients. Though the arthritis diagnoses were not clinically confirmed, the Swedish National Patient Register has been shown to have high diagnostic validity for rheumatic conditions. ${ }^{8}$ Malignancies were identified using the Swedish Cancer Register. Overall, the study did not reveal a significantly elevated cancer risk in biologics-naive patients with juvenile arthritis (RR 1.1, CI 0.9 to 1.5$)$ but the authors noted that the RR increased to 2.3 (CI 1.2 to 4.4 ) when analysing only patients from 1987 onward. This higher RR from 1987 onward was mainly driven by lymphoproliferative cancers. The authors speculated that the introduction of methotrexate may have contributed to this increased risk seen after 1987. Certainly, the cancers that occurred in our cohort arose in the years after 2000, but this was also the period over which $70 \%$ of our observation time occurred.

Comparisons between cancer risk in juvenile arthritis and RA are problematic because the two diseases are not the same. Juvenile arthritis includes a much wider range of disease, including many children who do not necessarily have widespread systemic inflammation and/ or exposure to the same drugs that adult patients with RA typically do. In general, many suspect that the risk of cancers such as lymphoma is likely linked to chronic active inflammation. Our study combined all patients with juvenile arthritis regardless of severity, and we did not have the necessary data, nor adequate power, to stratify the sample in order to study cancer risk only in the subgroup of children with very severe juvenile arthritis only.

Published data on drug effects and malignancy in juvenile arthritis are scarce, ${ }^{9-11}$ and drug-related analyses were not the specific aim of our current study. However, we did determine that six of the nine participants with cancer had a prior history of biologics and/or DMARDs (one of the three haematological malignancies, and four of the remaining six cancers had been exposed to biologics). We were unable to examine rheumatic disease activity, but we do note that within the distribution of arthritis types for the cancer cases, there does not seem to be an over-representation of the more severe juvenile arthritis types (such as polyarticular or systemic).

Our study provides unique data on a large number of patients with clinically confirmed juvenile arthritis, and our outcome was determined by the 'gold standard' of cancer registry linkage. One potential methodological limitation was that we may have incompletely accounted for patients who had been lost to follow-up or had moved out of the regional jurisdiction (province or state) where the cancer linkage was performed. This may have led to a conservative estimation of the SIR because of missed cancers (although likely very few) and some inflation of the observation time. However, this could have been a potential limitation in any of the currently published studies of cancer risk in juvenile-onset arthritis.

We also point out that, since the mean duration of follow-up was 6.8 years, few patients in our study were followed past early adulthood. This is much longer than most other published studies. Still, given the evidence linking disease activity and duration with increasing lymphoma risk in patients with RA, ${ }^{12}{ }^{13}$ further studies should investigate cancer risk in patients with juvenile arthritis in mid to late adulthood. Our study was unable to definitively comment on drug effects on cancer risk in juvenile arthritis and, given the sparse and conflicting data on this issue, targeted studies in this area are imperative. However, our study was consistent with our prior preliminary findings of no clear increased malignancy risk in other cohorts with clinically confirmed juvenile arthritis. ${ }^{14}$ Though ideally we would have liked to describe cancer incidence in relationship to arthritis type, this was difficult given the change in nomenclature over the years. Owing to the low number of cancers overall, we were unable to provide reliable estimates of cancer risk by arthritis type. Thus, if there is effect modification of cancer risk by arthritis type, we would need additional data in order to document or rule out such effects. This is, unfortunately, a limitation of all existing studies of cancer risk in juvenile arthritis.

\section{CONCLUSION}

In this analysis of the largest ever sample of patients with clinically confirmed juvenile-onset arthritis, using tumour registry linkage for outcome ascertainment, cancer was a relatively infrequent outcome. Three of the nine observed cancers were haematological. Longer follow-up of this population would be necessary to accurately capture cancer risk in the later adult years. Two-thirds of the cancer cases that we detected had been exposed to DMARDs and/or biologics, but it is not possible to infer a causal relationship between these exposures and cancer risk. Further study of drug effects is thus warranted. 
Author affiliations

${ }^{1}$ Department of Medicine, McGill University, Montreal, Quebec, Canada

${ }^{2}$ Department of Medicine, University of Calgary, Calgary, Alberta, Canada

${ }^{3}$ Northwestern University, Feinberg School of Medicine, Chicago, Illinois, USA

${ }^{4}$ Division of Rheumatology, The Hospital for Sick Children, Toronto, Ontario, Canada

${ }^{5}$ Department of Rheumatology, Seattle Children's Hospital, Seattle, Washington, USA

${ }^{6}$ Department of Pediatrics, University of Manitoba, Winnipeg, Manitoba, Canada

${ }^{7}$ Department of Pediatrics, Children's Hospital of Eastern Ontario, Ottawa, Ontario, Canada

${ }^{8}$ Department of Pediatrics, Royal University Hospital, Saskatoon,

Saskatchewan, Canada

${ }^{9}$ Division of Pediatric Rheumatology, Riley Hospital for Children, Indianapolis, Indiana, USA

${ }^{10}$ Department of Pediatric Rheum San Francisco, University of California, San Francisco, California, USA

${ }^{11}$ Department of Pediatrics, Duke University Medical Center, Durham, North Carolina, USA

${ }^{12}$ Division of Clinical Epidemiology, Research Institute of the McGill University Health Centre, Montreal, Quebec, Canada

Acknowledgements This work was made possible by operating grant funds from the Canadian Institutes of Health Research and the National Institutes of Health.

Funding National Cancer Institute.

Competing interests None declared.

Ethics approval McGill University Health Centre. All participating centres obtained ethics approval.

Provenance and peer review Not commissioned; externally peer reviewed.

Data sharing statement No additional data are available.

Open Access This is an Open Access article distributed in accordance with the Creative Commons Attribution Non Commercial (CC BY-NC 4.0) license, which permits others to distribute, remix, adapt, build upon this work noncommercially, and license their derivative works on different terms, provided the original work is properly cited and the use is non-commercial. See: http:// creativecommons.org/licenses/by-nc/4.0/

\section{REFERENCES}

1. Lovell DJ. Juvenile idiopathic arthritis: clinical features. In: Kippel JH, Stone JH, Crofford LJ, White PH, eds. Primer on the rheumatic diseases. 13th edn. New York: Springer Science, 2008:142-5.

2. Beukelman T, Haynes K, Curtis JR, et al. Rates of malignancy associated with juvenile idiopathic arthritis and its treatment. Arthritis Rheum 2012;64:1263-71.

3. Setoguchi S, Solomon DH, Weinblatt ME, et al. Tumor necrosis factor alpha antagonist use and cancer in patients with rheumatoid arthritis. Arthritis Rheum 2006;54:2757-64.

4. Nordstrom BL, Mines D, Gu Y, et al. Risk of malignancy in children with juvenile idiopathic arthritis not treated with biologic agents. Arthritis Care Res (Hoboken) 2012;64:1357-64.

5. Kok VC, Horng JT, Huang JL, et al. Population-based cohort study on the risk of malignancy in East Asian children with juvenile idiopathic arthritis. BMC Cancer 2014;14:634.

6. Thomas E, Brewster DH, Black RJ, et al. Risk of malignancy among patients with rheumatic conditions. Int J Cancer 2000;88: 497-502.

7. Simard JF, Neovius M, Hagelberg S, et al. Juvenile idiopathic arthritis and risk of cancer: a nationwide cohort study. Arthritis Rheum 2010;62:3776-82.

8. Waldenlind K, Eriksson JK, Grewin B, et al. Validation of the rheumatoid arthritis diagnosis in the Swedish National Patient Register: a cohort study from Stockholm County. BMC Musculoskelet Disord 2014;15:432.

9. Diak P, Siegel J, La Grenade L, et al. Tumor necrosis factor alpha blockers and malignancy in children: forty-eight cases reported to the food and drug administration. Arthritis Rheum 2010;62: 2517-24.

10. McCroskery P, Wallace CA, Lovell DJ, et al. Summary of worldwide pediatric malignancies reported after exposure to etanercept. Pediatr Rheumatol Online J 2010;8:18.

11. Hooper M, Wenkert D, Bitman B, et al. Malignancies in children and young adults on etanercept: summary of cases from clinical trials and post marketing reports. Pediatr Rheumatol Online $J$ 2013;11:35

12. Baecklund E, lliadou A, Askling J, et al. Association of chronic inflammation, not its treatment, with increased lymphoma risk in rheumatoid arthritis. Arthritis Rheum 2006;54 692-701.

13. Hellgren K, Smedby KE, Feltelius N, et al. Do rheumatoid arthritis and lymphoma share risk factors? A comparison of lymphoma and cancer risks before and after diagnosis of rheumatoid arthritis. Arthritis Rheum 2010;62:1252-8.

14. Bernatsky S, Rosenberg AM, Oen KG, et al. Malignancies in juvenile idiopathic arthritis: a preliminary report. $J$ Rheumatol 2011;38:760-3. 